\title{
Physicochemical characterization and mechanical performance analysis of biaxially oriented PLA/PCL tubular scaffolds for intended stent application
}

\author{
Pooja Bhati ${ }^{1,2}$ - Ramya Ahuja ${ }^{1} \cdot$ Alok Srivastava $^{1} \cdot$ Pankaj $^{3} \cdot$ Shweta Singh $^{1} \cdot$ Priya Vashisth $^{1} \cdot$ Naresh Bhatnagar $^{1}$
}

Received: 23 July 2020 / Accepted: 29 October 2020 / Published online: 26 November 2020

(c) Springer Nature Switzerland AG 2020

\begin{abstract}
Polylactic acid (PLA) and its blends are potential materials for bioresorbable vascular tubular scaffold (TS) manufacturing. The usual method for TS fabrication for intended stent application includes firstly extruding the polymeric tube and then subjected to post-processing operations for tailoring its mechanical properties. In this study, PLA/PCL blend TS was fabricated by using a novel and facile fabrication method, which eliminates the much-needed post-processing operation on the extruded TS. Two different ways were used to extrude the TS for a comparative study; (a) conventional Extrusion process (SE), and (b) novel single-step Biaxial Expansion during Extrusion (BAE). FTIR spectra were used to investigate the detailed physicochemical evaluation of the TS. Mechanical studies show BAE TS exhibits better mechanical performance as compared to SE TS. Also, BAE PLA/PCL blend TS containing $5 \mathrm{wt} \% \mathrm{PCL}$ withstood higher burst pressure among the tested specimens. The water contact angle (CA) of BAE8\% TS is observed to be $71^{\circ}$ as compared to $81^{\circ}$ for the SE0\% TS. Further, BAE PLA/PCL TS scaffolds showed better cell viability by MTT assay as compared to pristine PLA.
\end{abstract}

Keywords Polylactic acid blends · Extrusion · Surface wettability · Mechanical testing · Biaxial expansion · FTIR

\section{Introduction}

Stents are class three medical devices used to open the constricted lumen of a vascular artery or peripheral vein to maintain proper blood flow. The advent of the metallic stents proved to be a better solution for opening an occluded artery than the balloon angioplasty due to its low restenosis rate and prevention of acute vessel closure. Researchers have suggested that the requirement of the stent is for a short duration until the artery healing and revascularization is complete. Though metallic stents have proved to be an effective solution for plagued arteryopening, but it has certain limitations. Stent thrombosis, late luminal enlargement, and late vessel remodeling, and requirement of prolonged anticoagulant therapy enlargement are some of the shortcomings which need to be taken care [1,2]. Bioresorbable Vascular Stent (BVS) has shifted the paradigm as it can provide support to the artery during the initial deployment period and get resorbed in the body after fulfilling its intended purpose.

Apart from the metals, the bioresorbable polymers which are getting popularity for stent fabrication include polylactic acid (PLA), polyglycolic acid (PGA), polycaprolactone $(P C L)$ and blends of them. PLA is under research for more than a decade, and the interest in it is due to its high mechanical strength, bioresorbable nature, and longer degradation time [3, 4]. PLA and its blends with polymers like PGA, Polyhydroxyl butyrate (PHB), and PCL are still under research to further improve PLA [5-9] and

Pooja Bhati, pooja1380@gmail.com | 'Department of Mechanical Engineering, Indian Institute of Technology, New Delhi, India. ${ }^{2}$ Department of Mechanical and Automation Engineering, IGDTUW, Delhi, India. ${ }^{3}$ Centre for Biomedical Engineering, Indian Institute of Technology, New Delhi, India. 
to make it a better candidate for the medical applications in general.

PLA is also a key polymeric material for many other medical devices other than coronary stents like bone plates, bone screws, etc. $[5,6,10-12]$. Tailoring the mechanical properties of PLA polymer and its blend with other polymer is an area of research to make these materials more suitable for different applications including those for the medical use [12-14]. PCL is another promising bioresorbable biomaterial which is having a low glass transition temperature of $-60^{\circ} \mathrm{C}$ and is more ductile than PLA [15]. The blending of PLA with PCL is considered to cause a positive improvement in the mechanical properties of the PLA. Thermal, mechanical, and morphological studies of the PLA/PCL blends have been studied by different researchers $[6-8,16,17]$. Other researchers have also studied the various methods such as 3D printing, combined melt extrusion, and tube drawing method, dip coating for fabricating the tubular scaffolds/stents for the vascular application [18-20]. However, the detailed study of the effect of the fabrication process on the properties of the blend TSs for cardiovascular stent application is the need of the hour.

Polymers TS made by extrusion has inferior mechanical properties. Thus, post-production processing is inevitably required on the manufactured TS to enhance their mechanical properties, which is a classified technology of few pioneers in this domain [21]. Radial stiffness is one of the main mechanical properties which should be sufficiently high in a polymeric stent to withstand the radial compressive forces of an artery. The polymeric stent will have a high radial strength and stiffness only if the TSs from which it will get cut will also have the required mechanical properties. Researchers have developed various ways of increasing the radial stiffness or radial strength of the stent TSs by secondary post-processing methods after tube manufacturing [21-23].

In this work, polymer TSs were fabricated by melt extrusion and by using in house-made novel setup for single-step extrusion and biaxial expansion of the extruded TSs for bioresorbable stent application [24]. The focus is to understand the effect of different fabrication processes on the PLA and PLA/PCL blended TS during the extrusion process. Tensile and burst testing were performed to evaluate the influence of the fabrication process on the mechanical performance of TS. Further, SEM images of the tensile fractured surface of TS revealed a uniform distribution of PCL into the PLA matrix. A stent should also have a hydrophilic surface so that enhanced endothelization of the polymeric material can be achieved. The surface hydrophilicity and cellular viability of the extruded TSs were also investigated.

\section{Experimental and characterization}

\subsection{Materials}

PLA, Ingeo 4032D grade(density $1.24 \mathrm{~g} / \mathrm{cc}$ [25] with $6.4 \pm 0.3 \mathrm{MFI} \mathrm{g} / 10 \mathrm{~min}$ at $210^{\circ} \mathrm{C} / 2.16 \mathrm{~kg}, \mathrm{Mn}=90 \mathrm{~kg} /$ $\mathrm{mol}$ and $\mathrm{Mw}=207 \mathrm{~kg} / \mathrm{mol}$ [13] from Naturework LLC, USA was the base study material. PCL, CAPA 6800 was from Perstorp, the UK with Mw of $120 \mathrm{kDa}$ and melt flow rate of $3 \mathrm{~g} / 10 \mathrm{~min}$ [13] was the choice of material for reducing the brittleness of PLA in this study. For In-vitro cell culture studies, Dulbecco's Modified Eagle Medium (DMEM) procured from Thermo Fisher Scientific, (India) and NCCS Pune (India) provided the Mouse Fibroblast (L929) cell line.

\subsection{Preparation of blends}

The blends were prepared using a Co-rotating twin-screw extruder TSE (PRISM EUROLAB 16, Thermo Fisher Scientific) having a high shear screw configuration for better mixing. Two blends were prepared by varying the wt $\%$ of $P C L$ i.e. PLA/PCL5\% (5\% PCL, 95\% PLA) and PLA/PCL8\% (8\% $\mathrm{PCL}, 92 \% \mathrm{PLA}), \mathrm{SE0} \%$ is taken as control sample. Before the blends were prepared, the polymers were dried in a vacuum oven for $12 \mathrm{~h}$ at $50^{\circ} \mathrm{C}$ to maintain the moisture level below $250 \mathrm{ppm}$ from preventing the hydrolysis of the polymer during blending.

\subsection{Tubular scaffold fabrication}

As the blended polymers used are hygroscopic in nature, therefore before using them for extrusion of the TSs it must be dried to have less than $250 \mathrm{ppm}$ moisture and is maintained at this level to prevent the hydrolysis of the polymer during extrusion. PLA blends were dried in the vacuum oven $(-700 \mathrm{mmHg})$ at a temperature of $50^{\circ} \mathrm{C}$ for 12-16 $\mathrm{h}$. The TSs of the blended PLA/PCL blends were then extruded using an annular crosshead die and a single screw extruder (make Labtech). Two different methods are employed for the extrusions of the TS in this work, as mentioned below:

1. Extrusion (SE) is a conventional extrusion process in which a tubular profile is fabricated continuously using an annular die and a pulling mechanism utilizing a caterpillar belt.

2. Novel Biaxial expansion of the tube during the continuous extrusion process (BAE) [24]. This process where along with pulling in the axial direction, radial stretching is also provided just after the annular die 


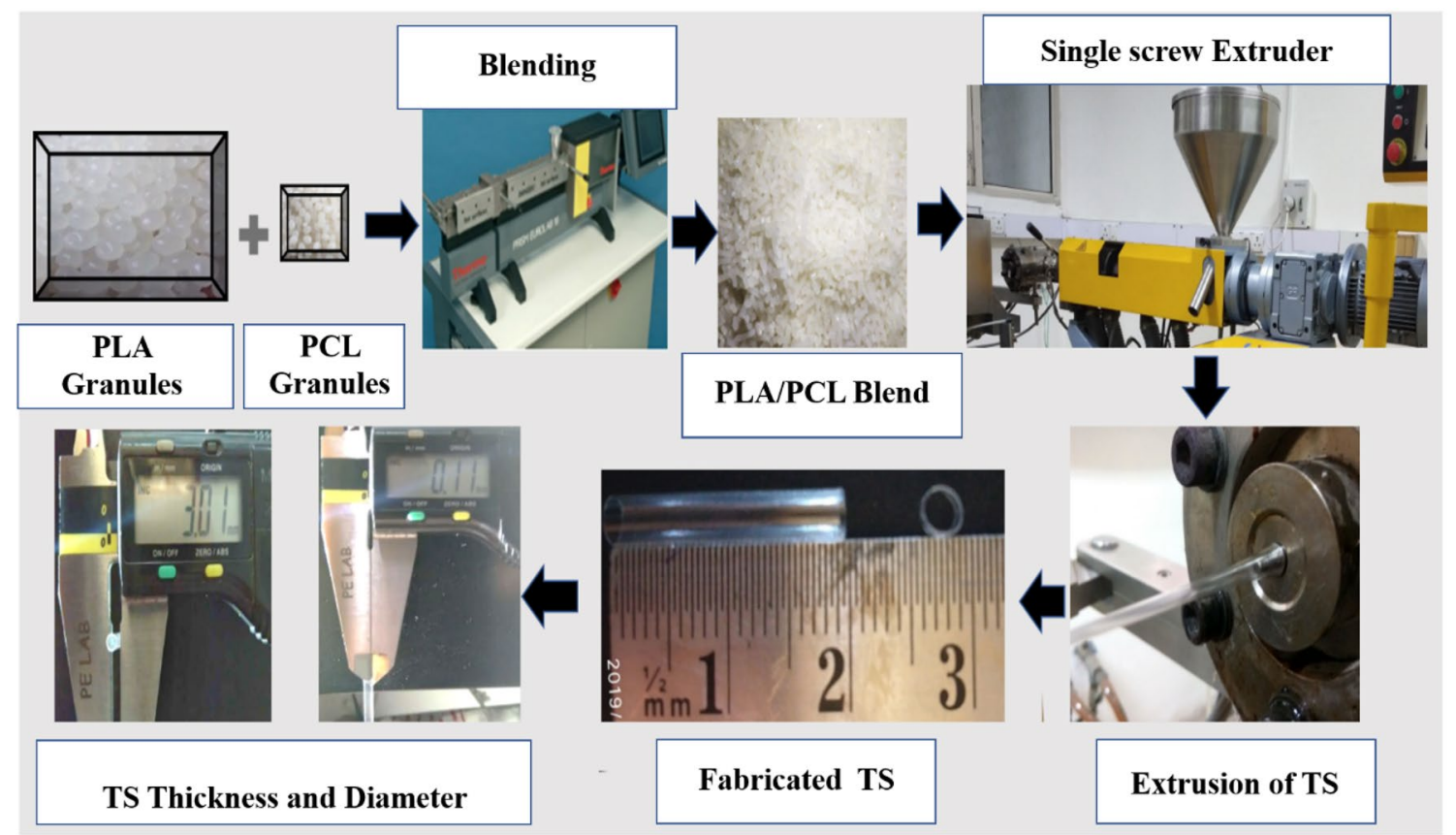

Fig. 1 Flow chart for TS fabrication process

exit. Figure 1 shows the flowchart for the TS fabrication process.

SE process is a conventional extrusion process for the tube production, whereas, for the BAE process, certain modifications were made in the traditional setup, and a custom setup was developed for the biaxial expansion of TS during the tube extrusion process, as shown in Fig. 2. Bhati et al. [26] have already reported that during the radial compressive testing, the biaxially expanded PLA/PCL5\% TSs have better radial strength as compared to pure PLA simply extruded TSs.

The processing parameter and other details related to the process are given in the Indian patent application [24].
All the extruded TSs having a diameter of $3 \pm 0.10 \mathrm{~mm}$ and thickness of $130 \pm 10 \mu \mathrm{m}$ were selected for the mechanical testing as these were the market standards for the coronary stent tubing at that point in time. The TSs extruded by simple extrusion method is designated as SE, followed by the percentage of PCL in it, e.g., if the tube is of PLA/PCL5\% blend and was simply extruded, then it is designated as SE5\% and the biaxially expanded TSs as BAE5\%.
Fig. 2 Schematic for biaxial expansion of the TSs during extrusion

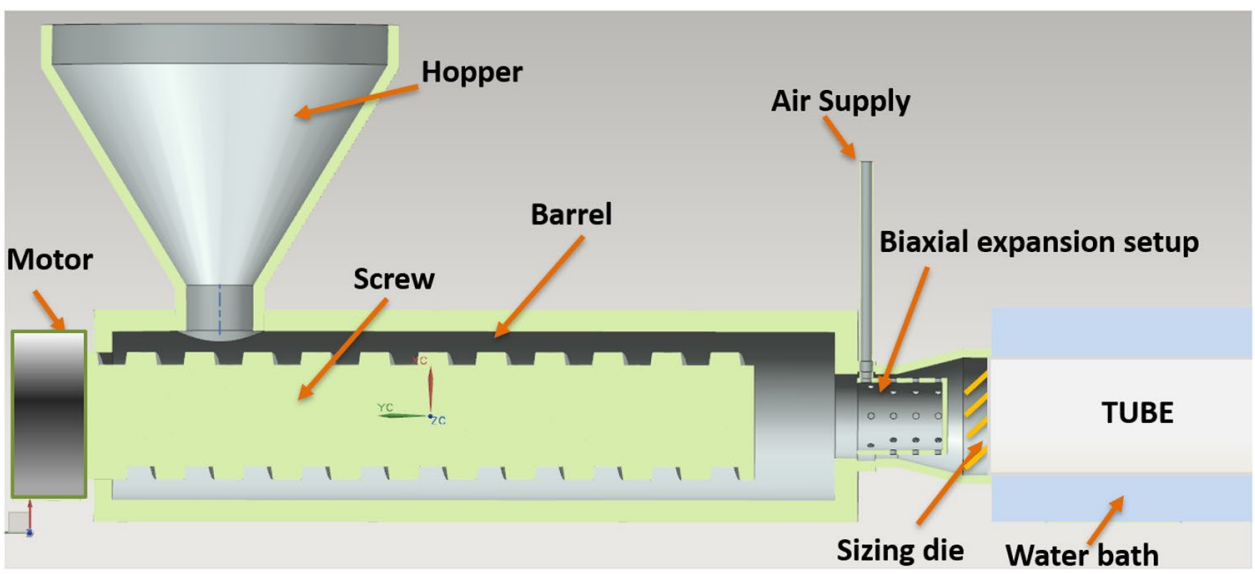

SN Applied Sciences A SPRINGER NATURE journa 


\section{Characterization of TS}

\subsection{Surface hydrophilicity}

Sessile drop method was used to determine the static contact angle on the TS surface. Deionized water droplets of $1 \mu \mathrm{L}$ volume were deposited on the samples. The images of the droplets on the samples were captured using a CMOS camera equipped with a magnifying lens at ambient temperature. The CA on the samples was evaluated by analyzing the images using Image $J$ software. An average of five readings at five different sites on the specimen is examined.

\subsection{FTIR analysis and chain orientation}

FTIR is a versatile tool that was used to identify the functional groups present in the material and for determining the molecular chain orientation in the polymer tube. The structural changes such as stretching or bending of the functional groups in the polymer due to the processing method could be evaluated by studying FTIR spectra. The functional groups present in the PLA and PLA/PCL blends were identified using the Nicolet iS50 FTIR spectrometer. The spectra were collected by averaging 32 scans with a resolution of $4 \mathrm{~cm}^{-1}$ using ATR technology. The chain orientation in the PLA and PLA/PCL blend TSs due to the two different fabricating method used were evaluated by studying the FTIR spectra in axial (parallel) as well as transverse (perpendicular) direction of tube axis to find peak position and peak intensities in the SE and BAE TSs which were investigated by studying the FTIR spectra [27].

\section{Mechanical properties}

\subsection{Tensile testing of the tubular scaffolds}

The tensile testing on the extruded TSs was performed as per ASTM D638. Subsequently, Young's modulus was calculated. The tensile testing on the extruded TSs was accomplished by making samples of $100 \mathrm{~mm}$ gauge length in the thickness range of $130 \pm 10 \mu \mathrm{m}$ and testing them on the Instron 5582 machine at a crosshead speed of $5 \mathrm{~mm} / \mathrm{min}$.

Five TSs in a sample group were tested, and their average value is reported. However, before testing, special grips for TS holding were designed and fabricated to ensure no-slip and no crushing of the scaffold ends happens during testing, and the failure of the TS is in the

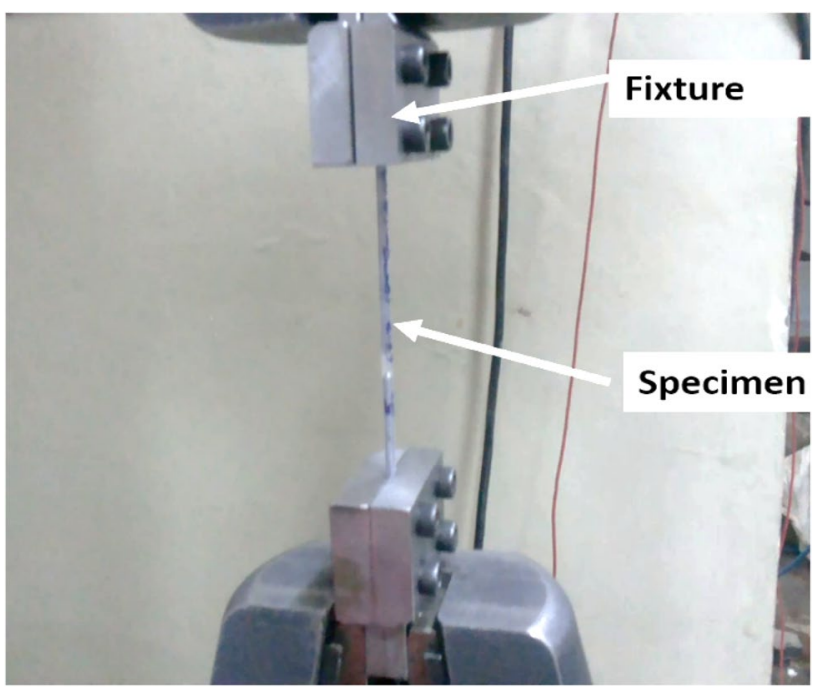

Fig. 3 Tensile testing of TSs using special fixtures on Instron

middle of the gauge length. Figure 3 shows the actual TSs during tensile testing.

\subsection{Burst pressure testing}

Burst pressure test was conducted as specified in ANSI 7198. The TSs of $50 \mathrm{~mm}$ length were pressurized from one end using compressed $\mathrm{CO}_{2}$ gas.

The pressure was increased at a rate of $0.5 \mathrm{bar} / \mathrm{s}$ until the tube bursting took place. The pressure at failure and change in tube diameter with the increase in tube pressure was recorded using a high-speed camera capturing $15,000 \mathrm{fps}$. Minimum five TSs in a sample group were tested to determine its mean burst pressure value. The schematic diagram and the actual setup of the burst test setup are shown in Fig. 4.

\section{Morphological characterization and cell studies}

The surfaces of the TSs after tensile fracture were examined under the SEM (Zeiss EVO 50) at accelerating voltages of 2-20 kV for determining the microstructural changes and for better understanding of the fracture surface morphology. The gold coating was applied on the fractured surface using BIO-RAD Polaran Sputter Coater.

Cytocompatibility of the extruded TSs was evaluated using MTT assay against L929 cells. In brief, cells were dispersed in Dulbecco's Modified Eagles Media (DMEM), which was supplemented with $10 \%$ fetal bovine serum (FBS) and seeded in 96 well plates at a density of $5 \times 10^{3}$ cells per well. After $72 \mathrm{~h}$ of incubation at $37{ }^{\circ} \mathrm{C}$ and $5 \%$ 
Fig. 4 (a) Schematic of Burst test setup developed in-house. (b) Actual setup during tube testing

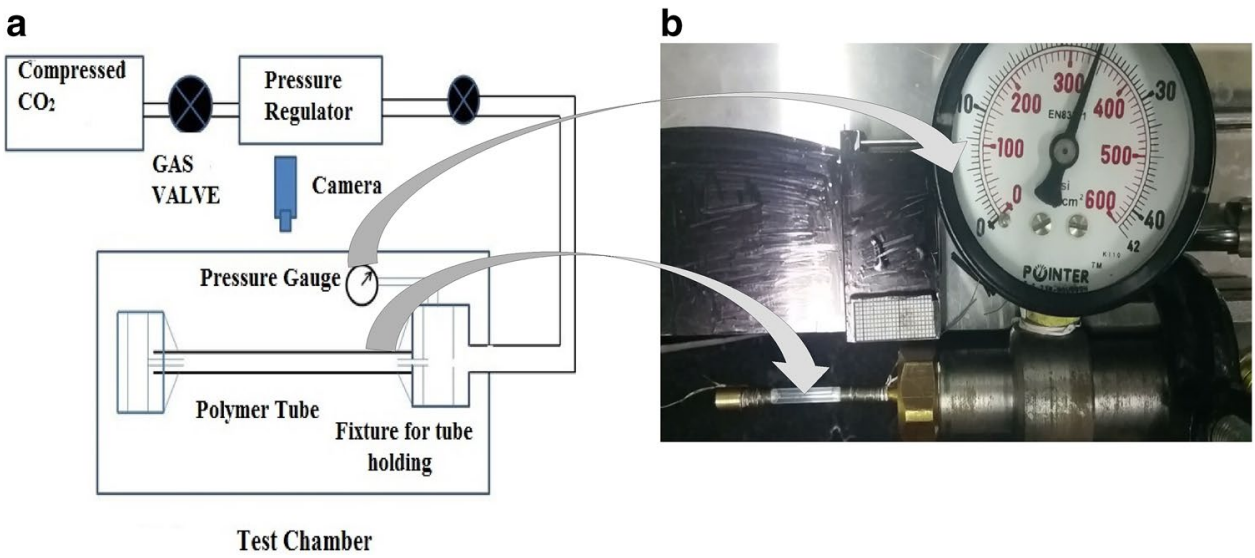

$\mathrm{CO}_{2}$, cell culture media were replaced with fresh media containing tube samples daily. Ten microliter of MTT solution $(5 \mathrm{mg} / \mathrm{mL})$ was added to each well and incubated for $3 \mathrm{~h}$ at $37^{\circ} \mathrm{C}$. Formazan crystals were dissolved in $200 \mu \mathrm{L}$ of DMSO, and absorbance of wells was recorded at $540 \mathrm{~nm}$ wavelength, using a microplate spectrophotometer make Powerwave XS2, Biotek Instruments, USA. All experiments were repeated three times.

Cell morphology on BAE5\% and SE0\% samples were examined using scanning electron microscopy. The samples were incubated for $24 \mathrm{~h}$ and carefully washed with PBS, then fixated with formaldehyde at $4{ }^{\circ} \mathrm{C}$ overnight. Afterward the samples were rinsed three times with PBS and sequential dehydration with alcohol was carried out for $10 \mathrm{~min}$ at different alcohol percentage $(30 \%, 40 \%$, $50 \%, 60 \%, 70 \%, 80 \%, 90 \%$, and $100 \%)$. This process was repeated two times. Further, the samples were dried in a desiccator. The samples were gold coated using BIO-RAD Polaran sputter coater and SEM images were taken (Zeiss EVO 50).

\section{Results and discussion}

The effect of the fabrication method and PCL percentage on the surface hydrophilicity was analyzed first on the bases of surface hydrophilicity. Materials were selected for further tensile and burst tests.

\subsection{Effect of fabrication method on surface hydrophilicity}

Re-reendothelialization of a TS is an essential factor for its proper functioning. A low water CA implies enhanced surface hydrophilicity or wettability of the material [28], which facilitates faster cell growth and thus is an important factor in it's in vivo functioning.

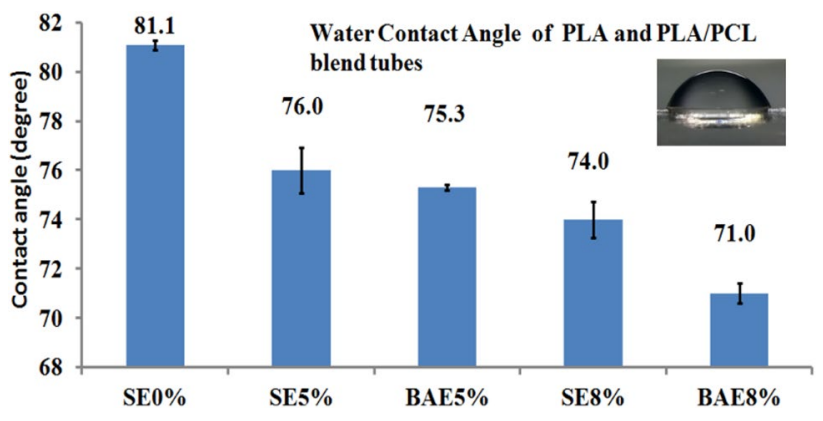

Fig. 5 Water contact angle of PLA and PLA/PCL blend TSs. Insight shows the DI water drop deposited on the tubular scaffold sample

Figure 5 shows the contact angle of DI water on different TSs of PLA and PLA/PCL blends extruded by simple extrusion (SE) and by biaxial extrusion (BAE) and the DI water drop deposited on the TS sample. PLA SE TSs show the highest $C A$ value, which is $81.07^{\circ}$, and the reported value of PCL CA is 71.1 [29]. It has been observed that as the percentage of $P C L$ is increased in the blend, $C A$ value shows a decreasing trend. Similarly, when the CA of the same blend was compared it is observed that water CA of the TSs extruded by the BAE fabrication process has a lower value as compared to SE TSs. This decrease in CA could be attributed to the biaxial stretching of the polymer chains during BAE process and a higher concentration of the carbonyl groups on the surface which increases the surface hydrophilicity of the biaxially extruded TSs and thus decrease the water $C A$ value.

Higher concentrations of carbonyl group on the BAE tube surface could have contributed to the decrease in the contact angle of the BAE TSs [30].

\subsection{FTIR and chain orientation}

FTIR spectrum is used to identify different functional groups present in any polymer or its blends. Each 
Table 1 Wavenumber and functional groups present in PLA

\begin{tabular}{ll}
\hline Wavenumber $\left(\mathrm{cm}^{-1}\right)$ & Functional group \\
\hline 1750 & $(\mathrm{C}=\mathrm{O})$ stretching \\
1450 & $(\mathrm{C}-\mathrm{H})$ stretching \\
1180 & $\mathrm{C}-\mathrm{C}(\mathrm{O})-\mathrm{C}$ stretching \\
1080 & $(\mathrm{C}-\mathrm{O})$ stretching \\
867 & $(\mathrm{C}-\mathrm{COO})$ stretching \\
\hline
\end{tabular}

functional group has a characteristic peak and peak position in the spectra as given in Table 1 [31]. FTIR can also be used for determining the chain orientation in the PLA, and PLA/PCL blends TSs. Any change in the peak intensity and peak position of a functional group can provide a deep insight into the structural change in the polymer or its blends. Figure 6a show the combined FTIR spectra of all the PLA and PLA/PCL SE and BAE samples and Fig. 6b shows the Individual material FTIR spectra peak for different extruded TSs. It is observed that the spectra for the BAE TSs have different peak intensity in the axial and transverse direction; however, SE TSs have relatively similar spectra intensity in both the directions. This difference in the peak intensity in BAE fabricated TSs is attributed to the biaxial stretching of the polymer chains as higher peak intensity means a higher concentration of functional group. The stretching of the polymer chain due to the processing method is further explained by studying the peak of the carbonyl group at $1752 \mathrm{~cm}^{-1}$. The higher the peak intensity, means more is the alignment of the PLA backbone [27]. The peak intensity for SEO\% TSs is similar for both the axial and transverse directions, and the same is the case with SE5\% and SE8\%.

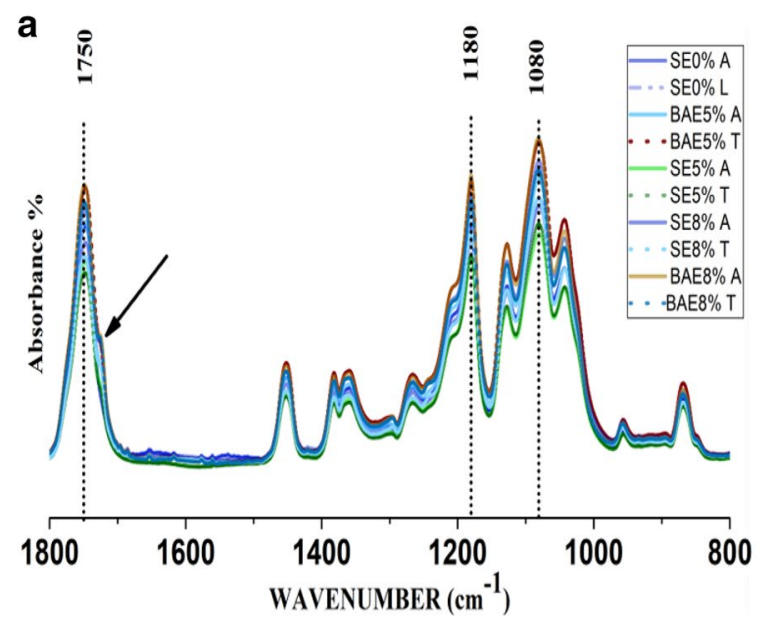

Along with $1752 \mathrm{~cm}^{-1}$ peak, the stretching is also observed at 1180 and $1080 \mathrm{~cm}^{-1}$ peaks, which shows the stretching of the other functional groups as well due to the novel fabrication method used. The TSs extruded by BAE methods show higher peak intensity in the transverse direction as compared to the axial direction, which indicates that the fabrication process causes a higher degree of chain alignment in the transverse direction for PLA/PCL blends TS.

The dichroic ratio of a particular band for the extruded TSs is expressed as $A_{a} / A_{T}$ where $A_{a}$ and $A_{T}$ are the absorbance intensities of the $C=O$ functional group in the axial and transverse direction, respectively and as the transverse alignment increases, the ratio decreases. The Dichroic ratio at $1752 \mathrm{~cm}^{-1}$ wavenumber of different extruded TSs is given in Fig. 7. It was observed that Dichroic ratio was lower for the TSs fabricated by BAE as compared to the SE method, and also with higher PCL percentage Dichroic ratio was decreasing. This peak is due to the addition of $P C L$ and representing the carbonyl stretching in $P C L$, and as $P C L$ percentage is increasing, the intensity of the

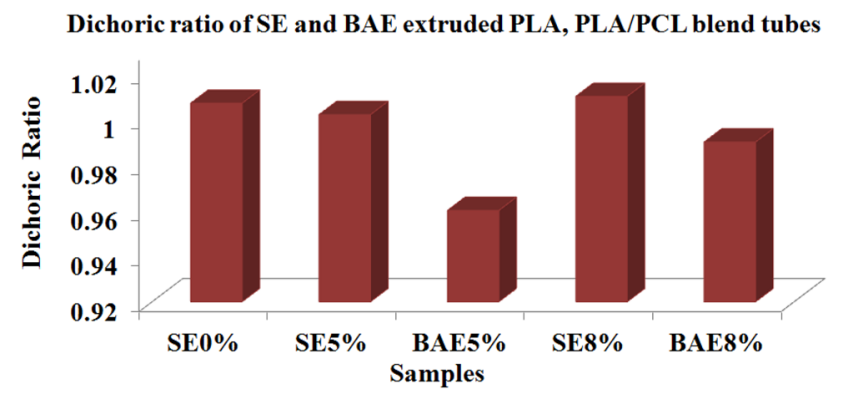

Fig. 7 Dichroic ratio of SE and BAE extruded PLA, PLA/PCL blend TSs

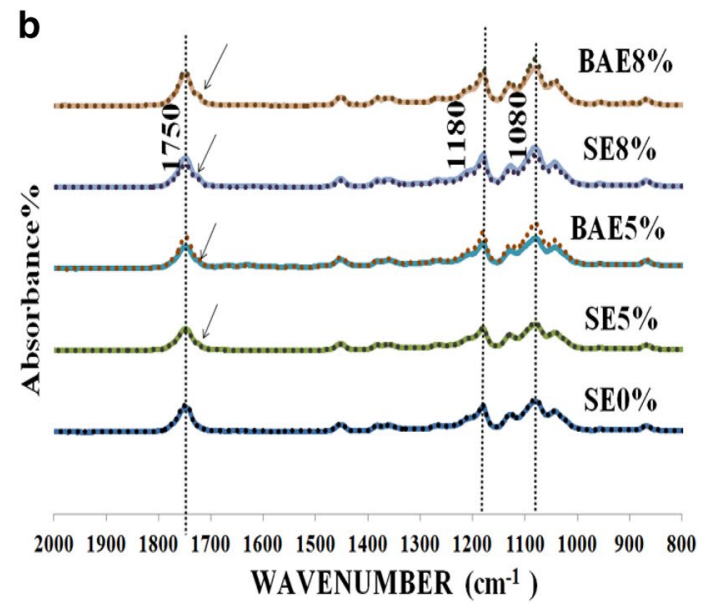

Fig. 6 (a) Show the combined FTIR spectra of the samples. (b) Individual FTIR-ATR Spectra of PLA and PLA/PCL blend TSs fabricated by SE and BAE methods in axial (solid line) and transverse direction (dash line) 
Fig. 8 (a) Average Young's modulus and (b) burst pressure comparison of PLA/PCL $5 \%$ and PLA/PCL $8 \%$ blend simply extruded and biaxially expanded TSs. $(*: p<0.05)$
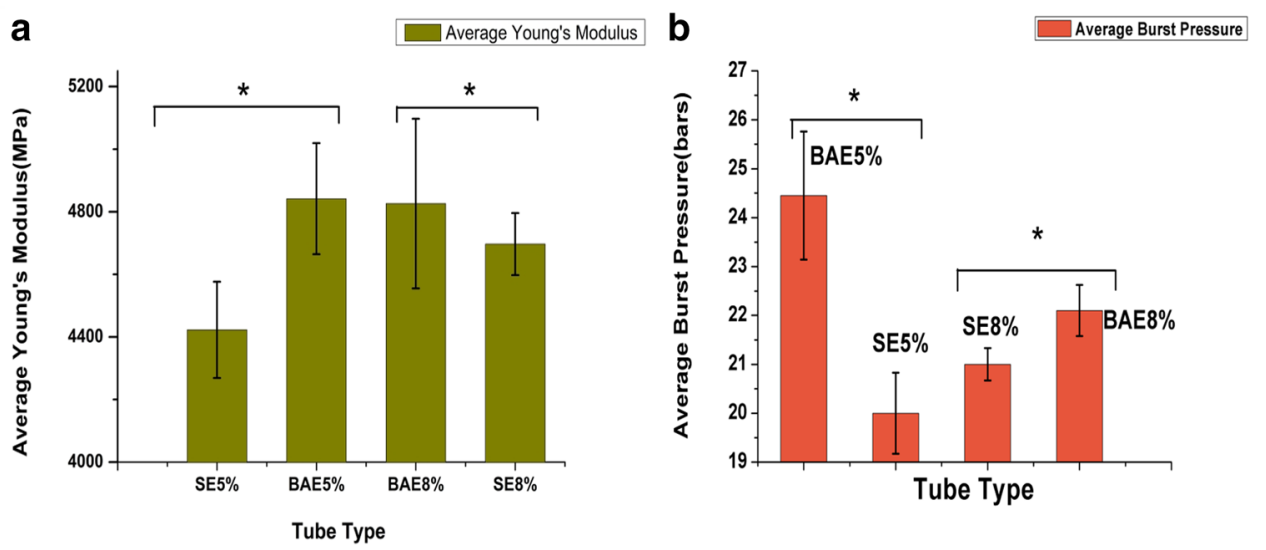

Table 2 Mechanical properties of PLA and PLA/PCL blend TSs

\begin{tabular}{lll}
\hline Sample & Tensile strength (MPa) & Toughness (MPa) \\
\hline SE0\% & $52.3 \pm 1.2$ & $3.3 \pm 2.8$ \\
SE5\% & $44.9 \pm 3.1$ & $4.3 \pm 2.4$ \\
BAE5\% & $46.2 \pm 2.4$ & $5.1 \pm 1.2$ \\
SE8\% & $49.7 \pm 0.5$ & $5.3 \pm 0.9$ \\
BAE8\% & $50.7 \pm 3.1$ & $8.3 \pm 3.1$ \\
\hline
\end{tabular}

shouldering peak is also increasing, which establish the homogeneity of the PLA/PCL blend, similar results were also reported by Khatri et al. [32].

\subsection{Tensile testing}

Only PLA/PCL TSs were studied for tensile testing as they showed better surface hydrophilicity as compared to SE0\% TSs. Figure 8a shows an average Young's modulus $(n=5)$ of the PLA/PCL TSs, which were extruded by different methods. In PLA/PCL 5\% blend, Young's modulus of TSs increases from 4.41 to $4.84 \mathrm{GPa}$ when the fabrication process changes from $\mathrm{SE}$ to $\mathrm{BAE}$.

Thus, BAE5\% TSs have 7.6\% higher Young's modulus value than the SE5\% TSs. Similar results were observed when SE8\% and BAE8\% TSs were compared. Young's modulus value increased to $4.82 \mathrm{GPa}$ from $4.62 \mathrm{GPa}$. However, the percentage increase in modulus Value is 4.32, which is smaller than the $5 \%$ blend TS. This more modest increase in modulus value could be due to the inherently lower Young's modulus value of PCL (500 MPa) [33] as compared to PLA. It indicates the method employed for the single-step biaxial expansion and extrusion of the tube is enhancing the mechanical properties of the extruded TSs to a significant level.

The higher Young's modulus values of the PLA/PCL TS as compared to PLA TS make the PLA/PCL TS better candidate for the intended vascular scaffold application. The higher value of Young's modulus can also be attributed to the polymer chain alignment due to the force applied by the pressurized air in the circumferential/radial direction and due to the longitudinal pulling forces in the axial direction. Thus, random polymer chains get oriented in an orderly fashion and closely packed. Hence increase in Young's modulus value. Table 2 shows the values of tensile strength and toughness of the extruded TSs.

\subsection{Burst strength}

For TS who are intended to be used in the arterial tree, Burst pressure is an important parameter. Burst Pressure provides an estimate of radial resistance provided by the TS to the applied internal pressure on its wall. Generally, the stent made from TS should maintain its strength in vivo after its deployment at the constricted artery site. The deployment of the stent at the constricted lumen is by dilating the balloon, and the material undergoes plastic deformation. SE5\%, BAE5\%, SE8\%, and BAE8\% are the TSs which were taken for a burst pressure study due to the better dichroic ratio and improved toughness of these samples as compared to SE0\%.

Figure $8 \mathrm{~b}$ shows the mean value of burst pressure in bars. It is evident from the figure that the mean burst pressure for the SE5\% is lowest, i.e., 20 bar. However, the mean burst pressure of BAE5\% TSs is 24.45 bars, which is $22.5 \%$ higher than the SE5\% TSs burst pressure value and is significantly different. The high burst pressure value of the $\mathrm{BAE} 5 \% \mathrm{TS}$ s is due to the radial resistance provided by the aligned polymeric chains in a circumferential direction.

Similarly, SE8\% and BAE8\% TSs show the same trend where BAE8\% TS is observed to have higher burst pressure value as compared to the SE8\% TSs. FTIR spectra of BAE TSs also confirmed the higher alignment of the polymer chain in the transverse direction as compared to their SE counterparts. This proves that the TSs extruded by the modified setup have better radial strength as 
compared to the TSs extruded by the conventional method. Thus, biaxial expansion during extrusion is a better method to be employed for TSs extrusion than the conventional extrusion process in case of the stent or medical tubing. A bioresorbable tubular Scaffold should have sufficient mechanical strength with minimum possible wall thickness to withstand in-vivo stress after its deployment, which is imposed on it by the local biological environment. It is of the utmost importance when the scaffold has to maintain its integrity and provide support to the diseased artery before bioresorbption.

\subsection{Morphological analysis of tensile fractured surfaces}

SEM micrographs of the tensile fractured surfaces of the PLA and PLA/PCL blends are shown in Fig. 9. There is miscibility between PLA and PCL as no cavities are present, and no separate phase of PCL is visible in the PLA matrix. Thus, phase miscibility and chain orientation is the primary reason for the improved tensile properties of the PLA/PCL blend TSs. Phase immiscibility of the PLA and PCL has already been reported by various researchers [34-37] in the past. However, no phase immiscibility is visible in the blends, which could be due to the novel manufacturing
Fig. 9 SEM images of the tensile fractured surfaces of PLA, PLA/PCL SE and BAE TSs. (a, b) SE0\% (control), (c, d) SE5\%, (e, f) $\mathrm{BAE} 5 \%,(\mathbf{g}, \mathbf{h}) \mathrm{SE} \%$ and $(\mathbf{i}, \mathbf{j})$ BAE $\% \%$

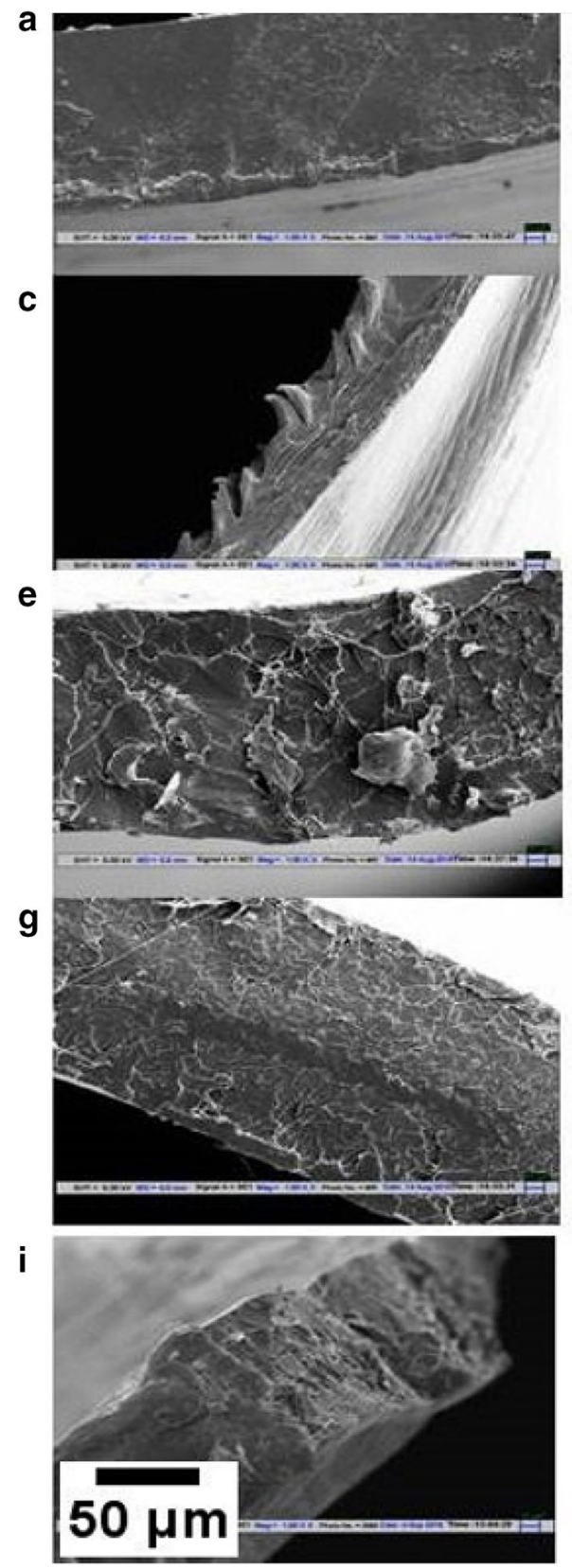

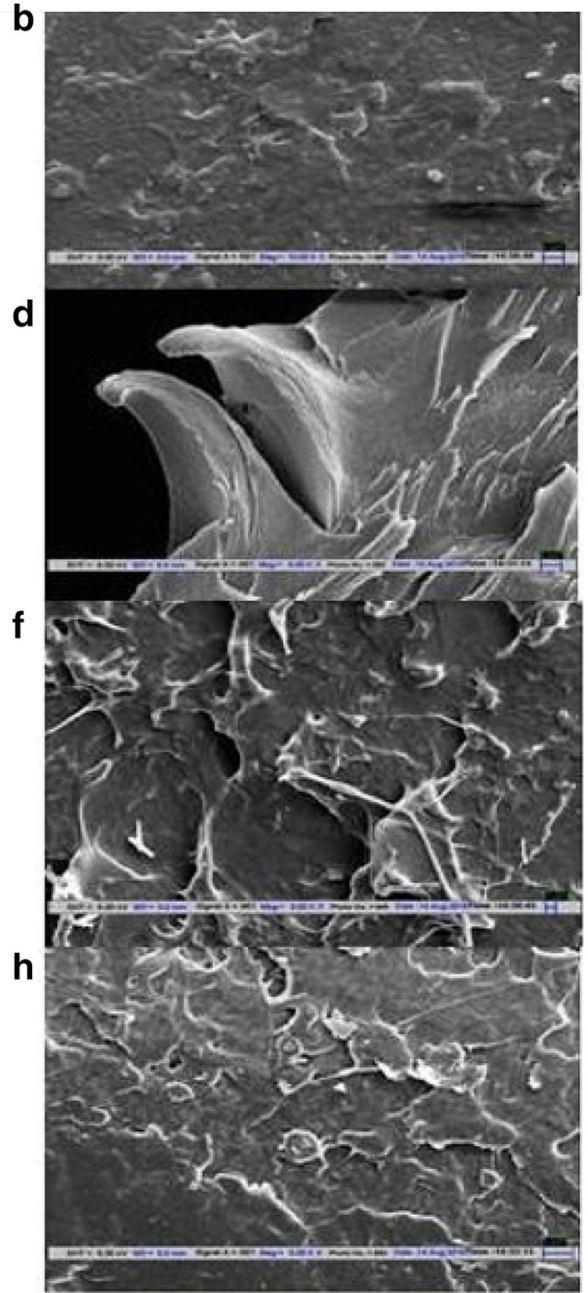

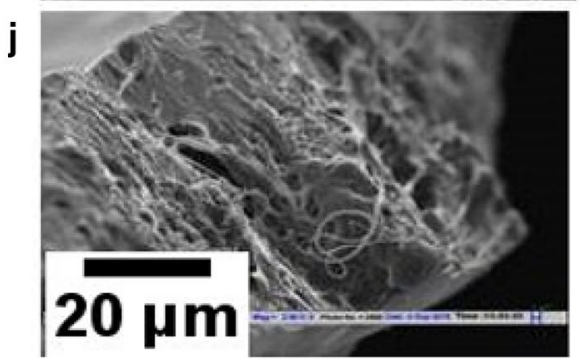



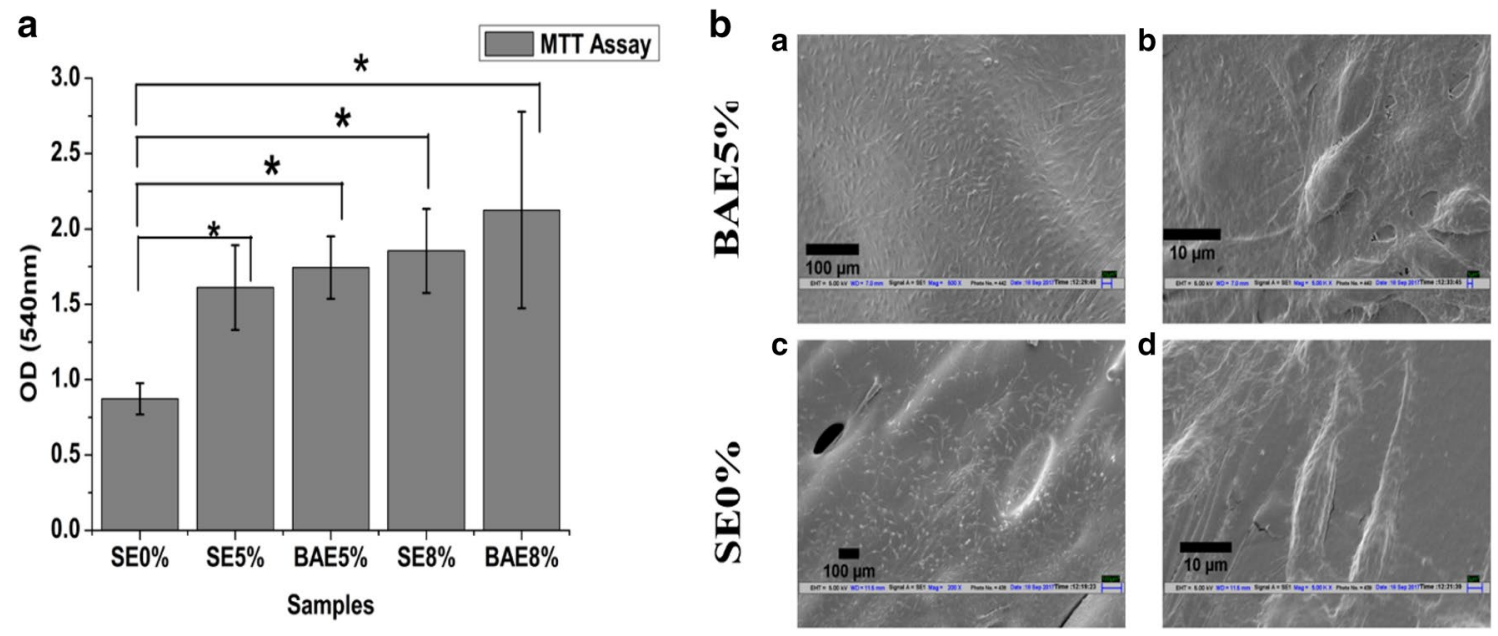

Fig. 10 (a) MTT assay of extruded TSs of PLA and PLA/PCL blends. SE0\% is taken as control. ( $\left.{ }^{*}: p<0.05\right)$. (b) SEM images of cultured L929 fibroblast on the BAE5\% $(a, b)$ and SEO\% $(c, d)$ after $24 \mathrm{~h}$ of cell seeding

method employed for tube extrusion. This result is in line with the result reported by Ostafinska et al. [38] for the PLA/PCL blend.

\section{Cytocomapatibilty studies}

\subsection{MTT assay}

This study is conducted to evaluate the cytocompatibility of the PLA/PCL blend tube. In Fig. 10a SE0\% was taken as a control sample. The TSs were seeded with L929 mouse fibroblast cells and were monitored for $72 \mathrm{~h}$ after cell seeding. From Fig. 10a, it can be inferred that the survival rate of the cells on the PLA/PCL blend TSs is better than the control sample and is apparently statistically different. The samples of BAE8\% had shown the highest cell survival rate and it differs from the control value. MTT study further proved that all the PLA/PCL blend samples are better material for cell growth as compared to the control material.

\subsection{Cell morphology}

SEM micrographs were used for assessing the cell morphology qualitatively. The SEM micrograph of the L929 fibroblast attached to BAE5\% and SE0\% TSs surface after $24 \mathrm{~h}$ are presented. Figure $10 \mathrm{~b}(\mathrm{a}, \mathrm{c})$ shows the homogenously covered BAE5\% and SEO\% TSs surface, respectively, and Fig. $10 b(b, d)$ shows the morphology of the fibroblast cultured on the TS surfaces. It shows that after $24 \mathrm{~h}$, cell proliferation was superior on biaxially expanded PLA/PCL $5 \%$ TS as compared to the pure PLA. It could also be due to increased hydrophilicity of the blended TS as compared to pristine PLA.

\section{Conclusions}

The main objective of this work was to establish a continuous fabrication process for manufacturing of bioresorbable polymeric TSs, which enhances the mechanical properties as compared to the conventional extrusion process.

The PLA/PCL blend TSs were successfully produced by BAE developed in the house [24], that exhibits enhanced mechanical and surface hydrophilicity. The chain alignment and molecular backbone stretching are confirmed by the FTIR spectra. PCL has a synergetic effect on the blend. The optimum PCL percentage for the blend was observed to be $5 \%$.

BAE5\% TSs are having improved overall properties along with better cell proliferation compared to others; hence, it could be concluded that BAE method improves the mechanical performance of TS and BAE5\% TSs is a potential candidate for intended stent Application.

Acknowledgments We acknowledge the Central Research facility (CRF) and Nanoscale Research Facility (NRF) at IIT, Delhi for the instrumentation facility used in this work.

Conflict of interest On behalf of all authors, the corresponding author states that there is no conflict of interest.

\section{References}

1. Serruys PW, Garcia-Garcia HM, Onuma Y (2012) From metallic cages to transient bioresorbable scaffolds: change in 
paradigm of coronary revascularization in the upcoming decade? Eur Heart J 33:16-25b. https://doi.org/10.1093/eurhe artj/ehr384

2. Onuma Y, Ormiston J, Serruys PW (2011) Bioresorbable scaffold technologies. Circ J 75:509-520. https://doi.org/10.1253/ circj.CJ-10-1135

3. Mani G, Feldman MD, Patel D, Agrawal CM (2007) Coronary stents: a materials perspective. Biomaterials 28:1689-1710. https://doi.org/10.1016/j.biomaterials.2006.11.042

4. Agrawal C, Haas K, Leopold D, Clark H (1992) Evaluation of poly(L-lactic acid) as a material for intravascular polymeric stents. Biomaterials 13:176-182 http://www.ncbi.nlm.nih. gov/pubmed/1567942

5. Grabow N, Bünger CM, Schultze C, Schmohl K, Martin DP, Williams SF, Sternberg K, Schmitz K-P (2007) A biodegradable slotted tube stent based on poly(L-lactide) and poly(4-hydroxybutyrate) for rapid balloon-expansion. Ann Biomed Eng 35:2031-2038. https://doi.org/10.1007/s1043 9-007-9376-9

6. Chen C, Chueh J, Tseng H, Huang H, Lee S (2003) Preparation and characterization of biodegradable PLA polymeric blends. Biomaterials 24:1167-1173

7. Tsuji H, Mizuno A, lkada Y (1998) Blends of aliphatic polyesters. III. Biodegradation of solution-cast blends from poly(L-lactide) and poly( $\varepsilon$-caprolactone). J Appl Polym Sci 70:2259-2268. https ://doi.org/10.1002/(SICI)1097-4628(19981212)70:11<2259::AIDAPP20>3.0.CO;2-J

8. Tsuji H, Ikada Y (1996) Blends of aliphatic polyesters. I. Physical properties and morphologies of solution-cast blends from poly(DL-lactide) and poly(e-caprolactone). J Appl Polym Sci 60:2367-2375 http://www.scopus.com/inward/recor d.url?eid=2-s2.0-0030173397\&partner|D=tZOtx3y1

9. Venkatraman S, Poh TL, Vinalia T, Mak KH, Boey F (2003) Collapse pressures of biodegradable stents. Biomaterials 24:2105-2111. https://doi.org/10.1016/S0142-9612(02)00640-3

10. Zilberman M, Nelson KD, Eberhart RC (2005) Mechanical properties and in vitro degradation of bioresorbable fibers and expandable fiber-based stents. J Biomed Mater Res B Appl Biomater 74:792-799. https://doi.org/10.1002/jbm.b.30319

11. Shafer BL, Simonian PT (2002) Broken poly-L-lactic acid interference screw after ligament reconstruction. Arthroscopy 18:1-4. https://doi.org/10.1053/jars.2002.32197

12. Siqueira DD, Luna CBB, Araújo EM, Ferreira ESB, Wellen RMR (2019) Biocomposites based on $\{P C L\}$ and macaiba fiber. Detailed characterization of main properties. Mater Res Express 6:95335. https://doi.org/10.1088/2053-1591/ab3496

13. Bai H, Xiu H, Gao J, Deng H, Zhang Q, Yang M, Fu Q (2012) Tailoring impact toughness of poly(L-lactide)/poly( $\varepsilon$-caprolactone) (PLLA/PCL) blends by controlling crystallization of PLLA matrix. ACS Appl Mater Interfaces 4:897-905. https://doi.org/10.1021/ am201564f

14. Siqueira DD, Luna CBB, Ferreira ESB, Araújo EM, Wellen RMR (2020) Tailored PCL/Macaíba fiber to reach sustainable biocomposites. J Mater Res Technol 9:9691-9708. https://doi. org/10.1016/j.jmrt.2020.06.066

15. Ulery BD, Nair LS, Laurencin CT (2011) Biomedical applications of biodegradable polymers. J Polym Sci B Polym Phys 49:832-864. https://doi.org/10.1002/polb.22259

16. Hiljanen-Vainio $M$, Varpomaa $P$, Seppälä J, Törmälä $P$ (1996) Modification of poly(L-lactides) by blending: mechanical and hydrolytic behavior. Macromol Chem Phys 197:1503-1523. https ://doi.org/10.1002/macp.1996.021970427

17. Guerra AJ, Cano P, Rabionet M, Puig T, Ciurana J (2018) 3D-printed PCL/PLA composite stents: towards a new solution to cardiovascular problems. Materials (Basel) 11:1-13. https:// doi.org/10.3390/ma11091679
18. Guerra AJ, San J, Ciurana J (2017) Fabrication of PCL/PLA composite tube for stent manufacturing. Proc CIRP 65:231-235. https://doi.org/10.1016/J.PROCIR.2017.03.339

19. Im SH, Park SJ, Chung JJ, Jung Y, Kim SH (2019) Creation of polylactide vascular scaffolds with high compressive strength using a novel melt-tube drawing method. Polymer (Guildf). https:// doi.org/10.1016/j.polymer.2019.01.067

20. Jia H, Gu S, Chang K (2018) 3D printed self-expandable vascular stents from biodegradable shape memory polymer. Adv Polym Technol 37:3222-3228

21. Kleiner LW, Oberhauser JP, Glauser T, Wrolstad DK, Wang Y (2014) Tube expansion processes for semicrystalline polymers to maximize fracture toughness. US8828305. http://www.google.co.in/ patents/US8828305

22. Al-Lamee K, Kelly A, Coates PD, Thompson GP, Caton-Rose P (2014) Method of producing a tube for use in the formation of a stent, and such tube. WO2014045068A1

23. Huang B, Wang Y (2009) Method to fabricate a stent having selected morphology to reduce restenosis. US 2009/0005860 A1

24. Bhatnagar N, Bhati P (2016) Polymer tubes for manufacturing stents. Indian patent Application No. 201611035281

25. NatureWorks (n.d.) Ingeo ${ }^{\mathrm{TM}}$ biopolymer 4032D technical data sheet biaxially oriented films-high heat, 1-3

26. Bhati $P$, Kumar A, Bhatnagar N (2016) Radial strength comparison of the PLA/PCL blends tubes manufactured by different fabrication processes. Front Bioeng Biotechnol. https://doi. org/10.3389/conf.FBIOE.2016.01.00580

27. Hossain KMZ, Parsons AJ, Rudd CD, Ahmed I, Thielemans W (2014) Mechanical, crystallisation and moisture absorption properties of melt drawn polylactic acid fibres. Eur Polym J 53:270-281. https://doi.org/10.1016/j.eurpolymj.2014.02.001

28. Rudolph A, Teske M, Illner S, Kiefel V, Sternberg K, Grabow N, Wree A, Hovakimyan M (2015) Surface modification of biodegradable polymers towards better biocompatibility and lower thrombogenicity. PLoS One 10:1-17. https://doi.org/10.1371/ journal.pone.0142075

29. Yeh C-C, Chen C-N, Li Y-T, Chang C-W, Cheng M-Y, Chang H-I (2011) The effect of polymer molecular weight and UV radiation on physical properties and bioactivities of PCL films the effect of polymer molecular weight and UV radiation on physical properties and bioactivities of PCL films. Cell Polym 30:261-276

30. Bhati P, Bhatnagar N (2017) Effect of processing parameters on surface hydrophilicity of porous PLA tubes prepared by gas assisted microcellular extrusion foaming technique. Mater Lett. https://doi.org/10.1016/j.matlet.2017.08.076

31. Smita Mohanty MC (2015) Effect of different solvents in solvent casting of porous caffolds-in biomedical and tissue engineering applications. J Tissue Sci Eng 06:1-7. https://doi. org/10.4172/2157-7552.1000142

32. Khatri Z, Nakashima R, Mayakrishnan G, Lee KH, Park YH, Wei K, Kim IS (2013) Preparation and characterization of electrospun poly( $\varepsilon$-caprolactone)-poly(l-lactic acid) nanofiber tubes. J Mater Sci 48:3659-3664. https://doi.org/10.1007/s10853-013-7161-8

33. Ebnesajjad S (ed) (2013) Plastic films in food packaging: materials, technology and applications. William Andrew, Norwich, NY

34. Todo M, Park S-D, Takayama T, Arakawa K (2007) Fracture micromechanisms of bioabsorbable PLLA/PCL polymer blends. Eng Fract Mech 74:1872-1883. https://doi.org/10.1016/j.engfracmec h.2006.05.021

35. Yeh J-T, Wu C-J, Tsou C-H, Chai W-L, Chow J-D, Huang C-Y, Chen K-N, Wu C-S (2009) Study on the crystallization, miscibility, morphology, properties of poly(lactic acid)/poly( $\varepsilon$-caprolactone) blends. Polym Plast Technol Eng 48:571-578. https://doi. org/10.1080/03602550902824390

36. Dell'Erba R, Groeninckx G, Maglio G, Malinconico M, Migliozzi A (2001) Immiscible polymer blends of semicrystalline 
biocompatible components: thermal properties and phase morphology analysis of PLLA/PCL blends. Polymer (Guildf) 42:7831-7840. https://doi.org/10.1016/S0032-3861(01)00269-5

37. Todo M, Takayama T (2011) Biomaterials_-physics and chemistry. InTech, Rijeka, Croatia. https://doi.org/10.5772/914

38. Ostafinska A, Fortelny I, Nevoralova M, Hodan J, Kredatusova J, Slouf M (2015) Synergistic effects in mechanical properties of PLA/PCL blends with optimized composition, processing, and morphology. RSC Adv 5:98971-98982. https://doi.org/10.1039/ c5ra21178f

Publisher's note Springer Nature remains neutral with regard to jurisdictional claims in published maps and institutional affiliations. 